\title{
A neutral wind instrument for nano-satellite platforms
}

L. J. Kordella, G. D. Earle, G. Roth, S. Noel, R. V. Robertson, R. L. Davidson, C. E. Holland, and V. Garg

Citation: Review of Scientific Instruments 89, 095001 (2018); doi: 10.1063/1.5054097

View online: https://doi.org/10.1063/1.5054097

View Table of Contents: http://aip.scitation.org/toc/rsi/89/9

Published by the American Institute of Physics

\section{Articles you may be interested in}

Calibration methods for the simultaneous measurement of the impulse, mass loss, and average thrust of a pulsed plasma thruster

Review of Scientific Instruments 89, 095103 (2018); 10.1063/1.5027047

An automated instrument for polarization-enhanced broadband nuclear quadrupole resonance (NQR) spectroscopy

Review of Scientific Instruments 89, 093106 (2018); 10.1063/1.5041002

A versatile apparatus for fermionic lithium quantum gases based on an interference-filter laser system

Review of Scientific Instruments 89, 093105 (2018); 10.1063/1.5045827

X-ray detector based on $\mathrm{Mn}$ doped $\mathrm{MgAl}_{2} \mathrm{O}_{4}$ and $\mathrm{Si}$ photodiode

Review of Scientific Instruments 89, 095104 (2018); 10.1063/1.5033504

FRAC: Fringing-RF-field-activated dc-to-pulse converter for low-energy ion beams

Review of Scientific Instruments 89, 095107 (2018); 10.1063/1.5023609

Dual energy-band excitation from a low power Rh anode X-ray tube for the simultaneous determination of low $\mathrm{Z}$ and high $\mathrm{Z}$ elements (Na-U) using total-reflection $\mathrm{X}$-ray fluorescence analysis (TXRF)

Review of Scientific Instruments 89, 093108 (2018); 10.1063/1.5044527
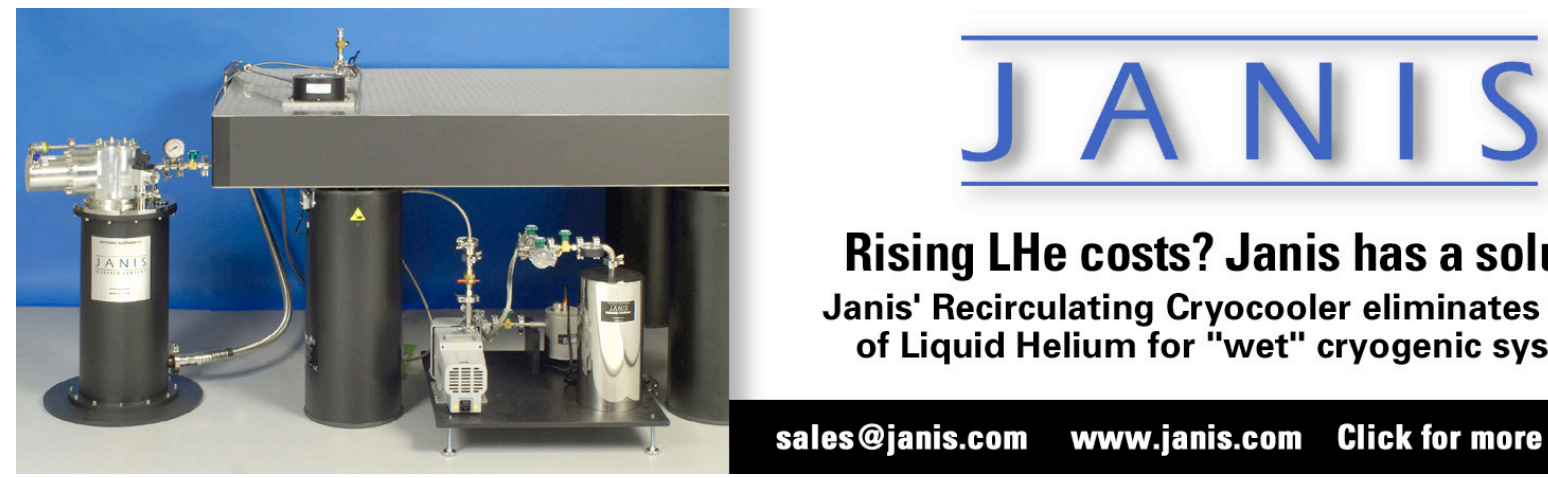

Rising LHe costs? Janis has a solution. Janis' Recirculating Cryocooler eliminates the use of Liquid Helium for "wet" cryogenic systems. 


\title{
A neutral wind instrument for nano-satellite platforms
}

\author{
L. J. Kordella, ${ }^{1, a)}$ G. D. Earle, ${ }^{1}$ G. Roth, ${ }^{2}$ S. Noel, ${ }^{1}$ R. V. Robertson, ${ }^{3}$ R. L. Davidson, ${ }^{4}$ \\ C. E. Holland, ${ }^{5}$ and V. Garg ${ }^{6}$ \\ ${ }^{1}$ Center for Space Science and Engineering Research, Virginia Tech, Blacksburg, Virginia 24060, USA \\ ${ }^{2}$ NAVAIR, Cherry Point, North Carolina 28533, USA \\ ${ }^{3}$ Cubic Aerospace, Reston, Virginia 20190, USA \\ ${ }^{4}$ Electrical and Computer Engineering Department, Utah State University, Logan, Utah 84322, USA \\ ${ }^{5}$ Sensor Systems Laboratory, SRI International, Menlo Park, California 94035, USA \\ ${ }^{6}$ Lutron Electronics, Allentown, Pennsylvania 18106, USA
}

(Received 14 April 2017; accepted 18 August 2018; published online 14 September 2018)

\begin{abstract}
Here we describe the first neutral wind sensor developed specifically for use on resource limited nano-satellite platforms. The instrument is a next generation redesign of the ram wind sensor flown on the Communications/Navigation Outage Forecasting System satellite for measurements of neutral velocity, temperature, and composition. Results of subsystem tests in vacuum conditions show lowpower operation, promising design, and good resolution of measured parameters over the operational pressure and energy ranges expected in the low Earth orbit environment. Published by AIP Publishing. https://doi.org/10.1063/1.5054097
\end{abstract}

\section{INTRODUCTION/MOTIVATION}

Virginia Tech has developed an in situ sensor called the Ram Energy Distribution Detector (REDD, pronounced red-dee) to measure the neutral density, changes in the neutral temperature, the light/heavy composition ratio, and the ram (in-track) velocity of the neutral gas in the reference frame of an orbital spacecraft. These are critically undersampled parameters for studying ion-neutral coupling effects that are relevant to a host of geophysical effects in Earth's thermosphere/ionosphere system, including scintillation and equatorial spread-F., ${ }^{1,2}$ Neutral density, temperature, and wind measurements are also critical parameters for understanding spacecraft drag in planetary atmospheres. Here we present the results of laboratory validation testing designed to characterize the performance of each REDD subsystem.

The operational concept of the REDD instrument is based on the Ram Wind Sensor (RWS) that was flown on the Communications/Navigation Outage Forecasting System (C/NOFS) satellite. ${ }^{3}$ REDD is a next generation neutral wind sensor designed for reduced size, weight, and power (SWaP) applications in the growing small satellite community.

\section{FUNCTIONAL DESCRIPTION OF THE RAM ENERGY DISTRIBUTION DETECTOR}

Figure 1 displays a cross section of the REDD device. The key components labeled include biased extraction plates near the aperture to reject background space plasma, an internal ionization source, a retarding potential analyzer (RPA) gridstack for particle diagnostics, and a high gain microchannel plate (MCP) detector for ion collection.

As the sensor name implies, the instrument aperture normal is oriented in the spacecraft ram direction. Because the satellite velocity in the low Earth orbit (LEO) altitude range

a)Electronic mail: lee8@ vt.edu of interest is much larger than both the atmospheric thermal velocity and any oblique wind components, the instrument admits the supersonic beam at near normal incidence to the aperture. This allows isolation of the atmospheric ram energy component for analysis.

For LEO altitudes, the influx atmosphere is partially ionized ( $\sim 1 \%$ plasma). In order to characterize the neutral component of the beam via electrical techniques, it is necessary to first remove these charged particles. This is achieved by means of electrically biased plates (referred to as the extraction plates) placed near the aperture (Fig. 1). Voltages applied to these plates create an electric field to repel and collect the negatively charged electrons and positive ions whilst leaving the neutral energy constituent largely unaffected.

The neutral beam passes the extraction plates and enters an ionization chamber where it encounters a controlled electron beam. Through collisions, a small fraction of the neutrals are ionized (about 1 in $10^{6}$ ) without significant perturbation to their energy distribution. This follows from conservation of energy and momentum for a worst case collisional interaction between an atmospheric neutral particle (e.g., $\mathrm{O}, \mathrm{N}_{2}$ ) and an electron. The transverse velocity gained by the neutral through the collisional ionization process can be estimated using conservation of energy and momentum and is less than $3 \%$ of the particle's total velocity. Furthermore this small transverse velocity component does not affect the passage of the newly created ion through the grid stack and thus does not contaminate the energy analysis. This transformation to ion form is performed to enable the RPA energy analysis technique. ${ }^{4}$ The newly created ions confront a swept positive polarity voltage (referred to as the retarding voltage or RV) in the grid stack that selectively rejects particles that lack sufficient energy to overcome the electric field. The fraction of the distribution that has enough energy to pass through the grids are measured as a current by a high gain (about $10^{6}$ ) MCP, thus compensating for the ionization efficiency loss. This measured current is correlated with the swept grid voltage as an $I V$ characteristic 


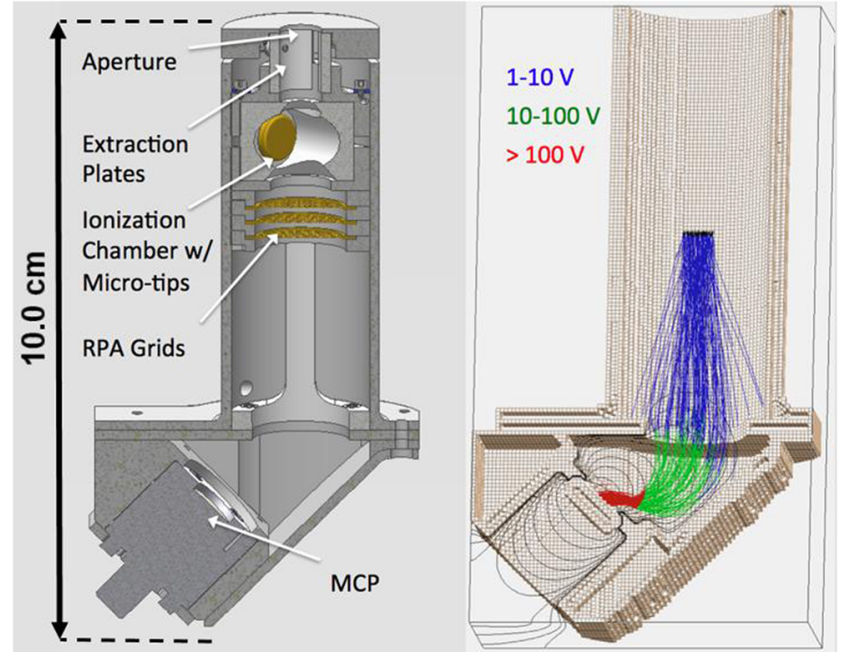

FIG. 1. (Left) A cross section of the REDD device with key components labeled. (Right) Results of a simulation study to trace ion trajectories form the grid stack to the front surface of the MCP. Detailed instrument specifications are shown in Table I.

that is subject to the RPA technique. ${ }^{4,5}$ Curve fitting techniques applied to the $I V$ characteristics provide measurements of the neutral particle density, temperature, velocity, and light/heavy ion species composition ratio. Figures 1-4 in the work of Fanelli et al. ${ }^{6}$ show example IV dependence on these physical parameters. The shape of the IV curve is affected by neutral temperature, allowing changes in the temperature to be identified.

In addition to its size, a key difference between the RWS and REDD sensors is the incorporation of a Spindt-type field emitter $^{7}$ as the REDD ionization source in place of the hot filament utilized by the RWS. Spindt-type field emitters consist of small, sharp, conductive cones that form a field electron source. The primary advantage of the micro-tip emitter solution as opposed to a hot filament electron source like that of the RWS is the reduction in power consumption within the ionization source by a factor of $\sim 1000$.

This improvement is highly advantageous for applications in the growing small satellite community. Spindt emitters have only been flown on sounding rockets so far, and in those tests,

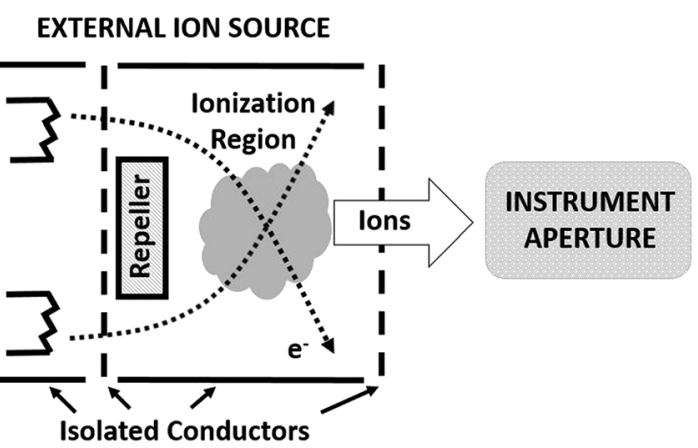

FIG. 2. A schematic view of the external ion source. Electrons generated by hot-cathode filaments collisionally ionize a fraction of the ambient neutral gas. Voltage biases are applied to conductive grids (dashed) and surfaces (solid) to control the trajectories and energy levels of electrons within the source in addition to producing an output ion beam.

they have proven to be functional in the relevant environment. ${ }^{8}$ The SRI International manufactured emitter selected for flight is specifically designed to withstand corrosion in atomic oxygen environments but has yet to be tested in long duration flight. An upcoming orbital mission will test these emitters as part of a technology demonstration instrument on the Lower Atmosphere/Ionosphere Coupling Experiment (LAICE) CubeSat. ${ }^{9}$

The expected operational altitude range of REDD is up to $\sim 600 \mathrm{~km}$ in the Earths atmosphere. In this range, it will encounter wind velocities of up to $\sim 600 \mathrm{~m} / \mathrm{s}$ and neutral temperatures from $\sim 1000$ to $1500 \mathrm{~K}$. The expected velocity sensitivity to changes between sequential measurements is on the order of $10 \mathrm{~m} / \mathrm{s}$ for RPA techniques ${ }^{10}$ with typical electrometer noise floors of $\sim 50 \mathrm{pA}$. Error associated with pointing is proportional to the cosine of the arrival angle. In order to achieve $\pm 10 \mathrm{~m} / \mathrm{s}$ precision, it requires misalignment less than $\pm 0.07^{\circ}$. This level of alignment is feasible for a three-axis stabilized cube satellite if a small star tracker is used.

\section{ELECTRO-MECHANICAL CONFIGURATION}

The sensor form factor presented in Fig. 1 is intended for integration with a cross-track wind sensor in a complete

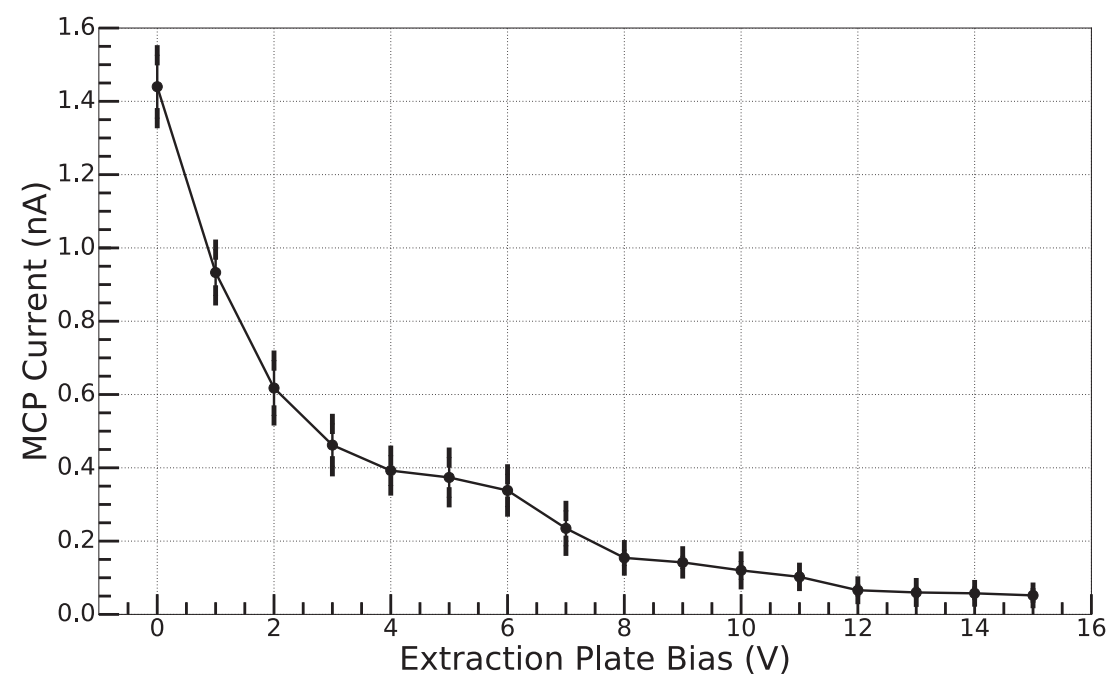

FIG. 3. MCP ion current plotted versus the extraction plate voltage at a fixed pressure of $0.1 \mathrm{mPa}$, which roughly corresponds to an altitude of $294 \mathrm{~km}$. For $V>15 \mathrm{~V}$, the ion flux is at the noise level of the instrument $(\sim 50 \mathrm{pA})$. 


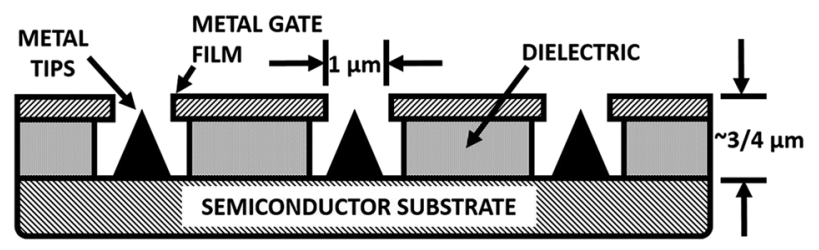

FIG. 4. Schematic of a Spindt cathode array. A potential is applied between the metal gate film and the metal tips to produce directed electron emission from the tips. Adapted with permission from Spindt et al., IEEE Trans. Electron Devices 38, 2355 (1991). Copyright 1991 IEEE. $^{16}$

TABLE I. REDD RWS SWaP comparison.

\begin{tabular}{lcc}
\hline \hline Parameter & RWS & REDD \\
\hline Volume $\left(\mathrm{cm}^{3}\right)$ & 3971 & 65 \\
Mass $(\mathrm{kg})$ & 1.95 & 0.116 \\
Power (W) & 2.84 & $1.06^{\mathrm{a}}$ \\
\hline \hline
\end{tabular}

${ }^{\mathrm{a}}$ Using prototype circuit boards that are subject to optimization.

$1 \mathrm{U}$ volume. The cross-track element is not discussed in this paper. It should be noted that the off-axis MCP geometry in Fig. 1 is intended to minimize the effect of photo and other secondary electrons. The right-side panel of Fig. 1 shows an example SIMION particle trajectory simulation to ensure that the vast majority of particles passing through the grids are collected by the detector due to the electric field generated by the high-voltage MCP. The slight beam spread seen here is due to preferential motion toward the grounded instrument body before encountering the MCP potential. The mechanical configuration of the REDD instrument can be tailored to conform to other space constraints. A comparison of the REDD SWaP with the RWS is shown in Table I. The RWS instrument details are extracted from internal C/NOFS documentation. Due to these characteristics, the REDD instrument is advantageously designed for small satellite applications.

\section{VACUUM TEST CONFIGURATION AND PROCEDURE}

Several stages of testing serve to verify the performance of the REDD device. In part, this is done to separately characterize major subsystems prior to higher-level system tests, but it is also necessary because neutral beam systems capable of flowing oxygen gas at hypersonic velocities in vacuum are not readily available. As a result, it has not yet been possible to perform true end-to-end testing on the REDD system. Instead, separate functional tests are performed to validate each subsystem, as described below:

1. Extraction plate testing: By mounting the REDD system in vacuum with an external ion source ${ }^{17}$, we can test the voltage level required on the extraction plates to ensure that no ambient ions or electrons enter the heart of the REDD instrument, where they can perturb the neutral gas measurements and/or contribute undesirable currents.

2. Emitter testing: The microtip emitters within the ionization chamber are tested to verify their integrity at LEO-level neutral densities to confirm that they produce stable emission currents and that they conform to FowlerNordheim emission characteristics. ${ }^{11}$

3. Ion source testing: The REDD sensor is mounted in vacuum, and the internal microtip-based ionization source is energized to verify that the MCP current scales as a function of the neutral chamber pressure. During this test, the REDD grids are biased to draw ions out of the ionization chamber since the neutral particles have no directed velocity after they are ionized. This test confirms the viability of the REDD ionization source over a range of pressures similar to the pressure at LEO altitudes. It also confirms the functionality and gain of the MCP sensor.

4. RPA testing: By injecting ions from an independent external ion beam source ${ }^{17}$ into the aperture of the REDD sensor, it is confirmed that the RPA grid stack within REDD is capable of measuring a current-voltage characteristic indicative of the incident ion energy distribution. Use of this external ion beam source acts as a proxy for a LEO-like energy neutral beam that would be ionized by REDD.

Figure 2 shows a schematic view of the external ion beam source used for these two tests. Electrons are generated using hot filaments like that of the RWS which are guided to undergo collisional ionization with the ambient neutral gas. Carefully selected voltage biases are applied to the components shown in Fig. 2 to guide the electron trajectories into desirable regions for ionization to satisfy the electron beam energy for most probable ionization and to output an ion beam spanning LEO-like energy magnitudes. Spatial variation of electron trajectories and ionization probability within the device leads to the creation of ions at different potential energies due to the surface biasing necessary to create the ion beam. The upper bound of the energy distribution is controlled by the surface labeled "Repeller" in Fig. 2. The positive voltage applied to this surface $(12 \mathrm{~V})$ guides the electrons to the central region of the device in addition to driving the generated ions outward toward REDD. The dependence of the energy distribution as a function of this repeller voltage is presented by Fanelli et al. ${ }^{6}$ This external ion source beam energy distribution of $0-12 \mathrm{eV}$ is constant for both utilized tests of the REDD extraction plates and the REDD RPA.

\section{RESULTS}

\section{A. Extraction plates}

Rudimentary particle trajectory modeling using the REDD sensor mechanical geometry shows that extraction plate potentials of roughly $\pm 15 \mathrm{~V}$ are sufficient to prevent all incident ions and electrons from penetrating into the sensor. It is important that the two extraction plates be held at opposite potentials so that an electric field exists between the plates. It is the $\Delta V$ between the plates that strongly influences the background charged particle trajectories and prevents admittance into the instrument. This effect is independent of moderate spacecraft charging.

Thirty trials of extraction plate voltage sweeps ranging from 0 to $12 \mathrm{~V}$ with simultaneous measurements of sensor 
throughput current (MCP detector current) confirm the function of the extraction plates. Figure 3 displays the results of this test at a fixed pressure corresponding to mid-range LEO altitudes.

It can be seen in the figure that the flux of ions through the extraction plates follows the desired suppression behavior with increasing voltage. For voltages above $\sim 12 \mathrm{~V}$, the MCP detects current plateaus at the electrometer noise level, which is $\sim 50 \mathrm{pA}$. These data confirm that a fixed extraction plate voltage greater than $12 \mathrm{~V}$ will remove ions from the incident beam so that only neutrals pass through to enter the ionization chamber. Since the electrons have less momentum than the ions, they are obviously removed by the equal and opposite voltage applied to the second extraction plate. Mitigation of minor spacecraft charging that could accelerate thermal ions is easily accomplished using the senpot technique described by Zuccaro and Holt. ${ }^{12}$

\section{B. Emitter testing}

Several tests justify the substitution of Spindt-type field emitters in place of a hot filament for the ionization source within the REDD sensor. The key investigatory parameters for these tests are the capability to produce electron beams of sufficient current density, energy, and stability to achieve the required ionization efficiency.

As previously described, the emitters consist of two closely spaced electrodes separated by a $\mathrm{SiO}_{2}$ dielectric: microtips (metal tips) and a forward counter electrode that we will refer to as the gate (metal gate film). This geometry can be seen in Fig. 4. The emitter tips are biased to a negative potential with respect to a gate electrode that acts to create a high electric field near the microtips. The resulting electron emission from metals under electric fields is characterized by the Fowler-Nordheim relationship. ${ }^{13}$ The theoretical current-voltage characteristic of a properly functioning emitter is given by

$$
I=V^{2} \Lambda \exp (-\kappa / V),
$$

where $\Lambda$ and $\kappa$ are constants that depend on the geometry of the emitters and the materials used to fabricate them, $I$ is the emitted electron current in amps, and $V$ is the potential difference in volts from the gate to tip. ${ }^{13}$ It is also key to note that the relationship is dependent on the potential difference between the two electrodes, and thus each may be floated relative to ground. Plots of $\ln \left(I / V^{2}\right)$ versus the inverse of $V$ produce a linear curve of negative slope, which is referred to as a Fowler-Nordheim characteristic. ${ }^{11}$ This is the standard way to visualize Spindt cathode performance. ${ }^{14}$

To avoid perturbations to the kinetic energy distribution of the newly ionized particles, it is important that the ions are created at the same potential energy. Variations in the initialized ion potential energies will lead to kinetic energy modulations that affect the distribution analysis. For this reason, the geometry shown in Fig. 4 is advantageous, in that the field generated by the microtips is shielded by the gate electrode. In the REDD application, the gate electrode is held at spacecraft ground, as is the ionization chamber. This ensures a uniform potential for all ions produced.

Figure 5 displays a Fowler-Nordheim curve generated using a Spindt-type emitter with microtips biased to roughly $-65 \mathrm{~V}$. In comparison, tungsten filament solutions such as that utilized by the RWS produce emission currents on the order of $\sim 100 \mu \mathrm{A}$. This is consistent with the emission values from the figure which range between $\sim 35 \mu \mathrm{A}$ and $\sim 155 \mu \mathrm{A}$. Though this curve can be extrapolated to a wider voltage range, these satisfactory emission current levels correspond to electron energies with adequate cross sections to ionize the neutral constituents in the LEO environment. It is also important to note the consistency of this $I V$ relationship, as is apparent from the small error bars.
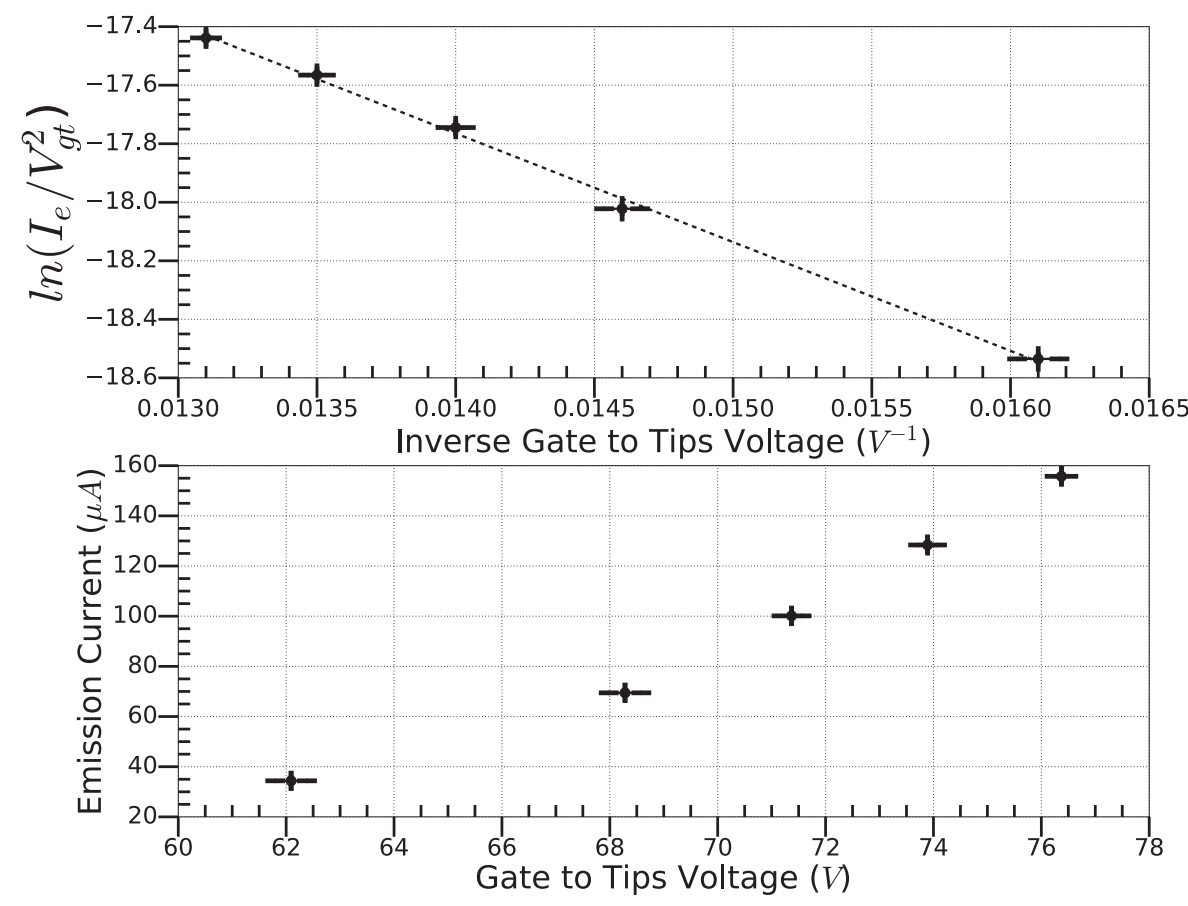

FIG. 5. A Fowler-Nordheim style plot (top) demonstrating emission characteristics of a Spindt-type field emitter sample with a linear best fit. Here $V_{g t}$ is the potential difference between the gate electrode and microtips in volts, and $I_{e}$ is the emission current in amps. The bottom panel shows the voltage current relationship. 


\section{Ionization source}

It is also necessary to confirm the compatibility of the microtips as a suitable ionization source for the REDD system geometry. Since we cannot yet flow a neutral beam into the aperture to test this in a realistic flow environment, we instead confirm that the microtip ionization source produces an ion density within the REDD system that scales with the neutral pressure in the vacuum chamber. Because of these stagnant conditions, we bias the RPA grids with small, increasingly negative potentials up to $-3 \mathrm{~V}$ to draw the newly created positive ions from the ionization chamber toward the MCP detector. Once through the gridstack, the particles are under the influence of the large electric field generated by the high voltage $(-2 \mathrm{kV}) \mathrm{MCP}$, which is sufficient to guarantee that they are collected. The grid voltages used for this specific subsystem test differs from the flight configuration where the standard RPA technique is implemented.

The data shown in Fig. 6 represent the variation in the ionization efficiency of the microtip emitter source as a function of the background neutral pressure in the vacuum chamber and hence the neutral density within the ionization chamber.
Because the emission capability of these microtip systems deteriorates at vacuum pressures near and above $\sim 1.3 \mathrm{mPa},{ }^{7}$ the most effective way to measure pressure fluctuations with the REDD system is to normalize the collected current by the current emitted from the microtips. This is accomplished in practice by connecting the isolated surface of the ionization chamber through an ammeter to the spacecraft ground. Though several data points in Fig. 6 are in this high pressure regime, this normalization retains the key quantity of ionization efficiency independent of the emission level and stability.

Two linear fits are shown: one for the low pressure range that coincides with flight pressures and a second for a higher pressure range that would not be encountered in a stable orbit. Further argument for the expected linear relationship is presented in Sec. VI. Based on the altitude range shown in Fig. 6, this will not be an issue during normal operation in the LEO environment, where the pressure inside the instrument will not exceed this limit until the latest stage of the spacecraft lifetime. This will occur at an altitude of $\sim 200 \mathrm{~km}$ based on the calculations presented in Sec. VI. At this altitude, because of the low ballistic coefficients, a small spacecraft will only survive for a short time before re-entry.
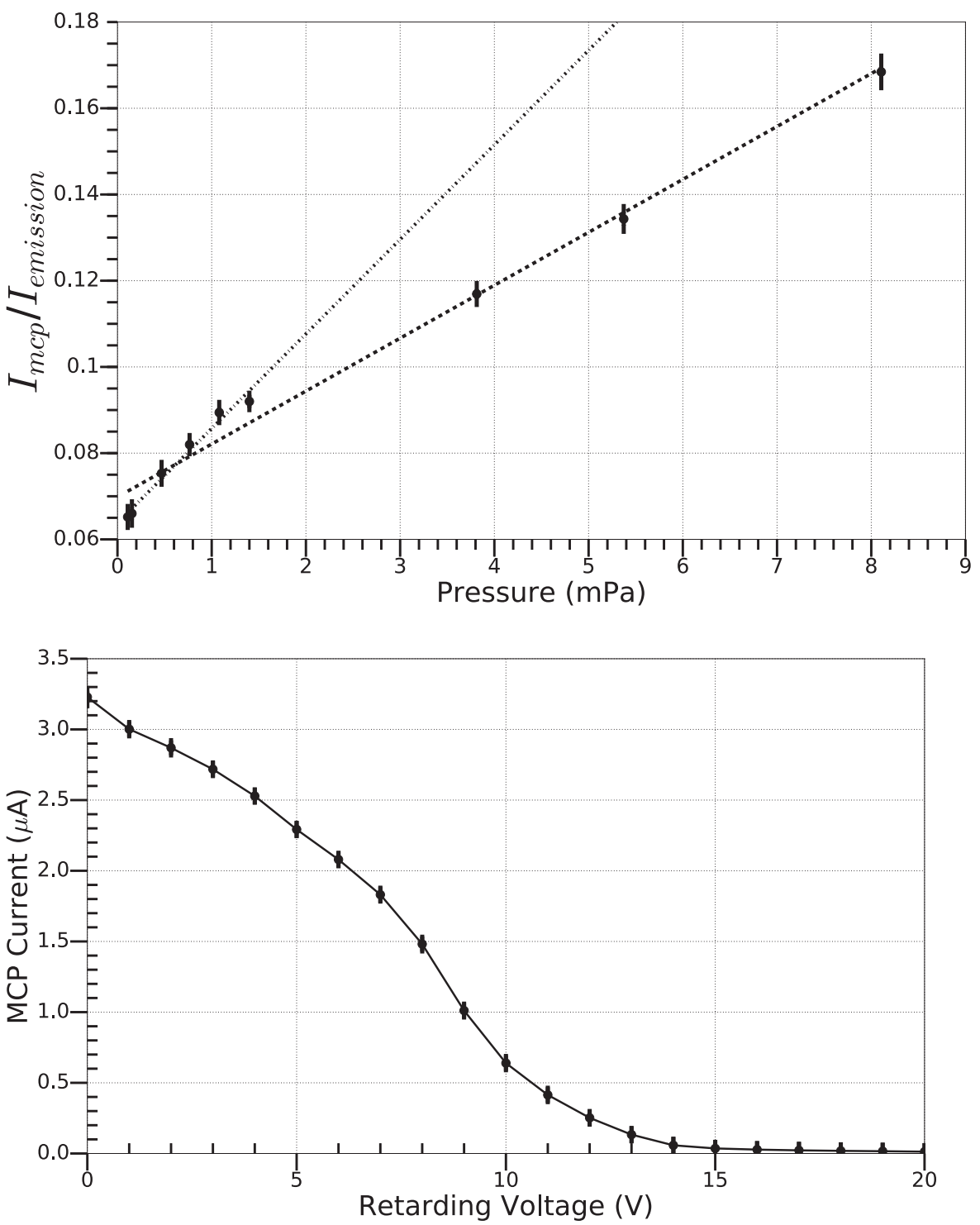

FIG. 6. The ratio of the current measured on the microchannel plate (MCP) to the microtip emitter current is plotted over a pressure range of $\sim 133 \mu \mathrm{Pa}$ to $\sim 8 \mathrm{mPa}$ of dry nitrogen gas. The error bars show the standard deviation over 30 independent tests.
FIG. 7. An RPA $I V$ curve generated in vacuum by placing an independent ion source in front of the REDD aperture and disabling the ion source and extraction plates within the REDD system. Note the very small error bars, indicating consistent performance over 30 independent tests. 


\section{Retarding potential analyzer}

The RPA gridstack in the REDD sensor is the key subsystem for analyzing changes in the neutral gas distribution function. The RPA technique has significant heritage for measuring ion distributions for in situ space measurements. ${ }^{4,6}$ The RWS was the first successful demonstration of this technique for neutral measurements. ${ }^{3}$ Many of the RWS design principles are common to the REDD sensor, which also includes improvements to the ionization source and form factor.

Validation of the RPA subsystem is accomplished using an independent ion source mounted in front of the REDD aperture. This source is operated to produce an ion beam with energies consistent with spaceflight conditions. The background chamber pressure is held in the $100 \mu \mathrm{Pa}$ range in agreement with LEO conditions.

Figure 7 shows the RPA $I V$ characteristic observed under the vacuum conditions listed above. The results show exceptional repeatability with minimal deviation over 30 trials.

\section{DISCUSSION}

The data presented here successfully validate each subsystem of the REDD instrument and establish some practical limits that improve its functionality. As shown in Fig. 3, extraction plate voltages of $\pm 15 \mathrm{~V}$ are sufficient to remove ions and electrons from the gas stream that enters the aperture. Desirable electron emission characteristics for the Spindt-type field emitters are shown in Fig. 5. The achievable flux levels (upwards of $100 \mu \mathrm{A})$, emission stability, and electron energies $(\sim 100 \mathrm{eV})$ for sufficient ionization cross sections are all key factors for implementation in the REDD instrument. However, special considerations need to be made in practice. Rigorous thermalvacuum preconditioning and contamination minimization of the emitter array are vital to maintain their performance. ${ }^{13}$ These ideal conditions are not easily achievable in a practical laboratory device that necessarily contains insulators and outgassing materials. By contrast, the space-borne orbital environment is advantageous as the instrument can undergo a long period of outgassing and numerous thermal cycles before being activated. Because of the potential fluctuations in emission when using microtips, it is important to measure the time varying emission currents continuously during use, so their variations can be accounted for during data analysis.

Figure 6 displays the sensitivity of the REDD instrument to variations in pressure. Linear fit curves are shown using both the full range of points and for points below $1.3 \mathrm{mPa}$ as a distinct cluster. A theoretical derivation for $\mathrm{MCP}$ detector current as a function of pressure for a single neutral species is shown below. Equation (2) shows the expression for the electronneutral collision frequency where $n_{n}, V_{e}$, and $\sigma_{n}\left(V_{e}, n_{\text {species }}\right)$ are the neutral number density, the electron speed, and the electron-neutral ionization cross section, which is a function of the electron energy and the neutral species, respectively,

$$
v_{e n}=n_{n} V_{e} \sigma_{n}\left(V_{e}, n_{\text {species }}\right) .
$$

Including the fundamental charge $e$, the MCP gain $G$, and an event probability function to model the likelihood that a collision produces ionization $P\left(V_{e}, n_{\text {species }}\right)$, we arrive at Eq. (3).
The relationship between the MCP detector current and the pressure in Eq. (3) is linear if the event probability function is linear. However, direct comparisons of Eq. (3) with the fits on Fig. 6 are difficult because of uncertainties in parameters like detector gain, ionization probability, electron-electron momentum transfer, and diffusive paths,

$$
I_{m c p}=p_{n} \frac{V_{e} \sigma_{n} e G P\left(V_{e}, n_{\text {species }}\right)}{k_{B} T_{n}} .
$$

The altitude range that corresponds to the pressures shown in Fig. 6 is dependent on the thermospheric conditions and a parameter referred to as the ram effect. Ram facing instruments with small aperture areas compared to the instrument chamber wall area act like a hohlraum. Entering gas molecules completely accommodate to the chamber walls before escaping, thus increasing the internal instrument pressure relative to the ambient exterior. ${ }^{15}$ This effect on the chamber pressure can be approximated by Eq. (3) from Hanson et al. 1992, here shown as Eq. (4) with the temperature T, the pressure P, the reciprocal of the most probable gas particle velocity $\beta$, the relative speed between the spacecraft and ambient gas $V$, and the angle between the aperture normal and the spacecraft velocity vector $\alpha$. Subscripts 0 and $g$ represent the external ambient and internal instrument quantities, respectively,

$$
P_{g} \approx 2 \sqrt{\pi} \beta V P_{0}\left(T_{g} / T_{0}\right)^{1 / 2} \cos \alpha .
$$

Considering the case of perfect instrument pointing in the ram direction $(\alpha=0)$ with an assumed instrument temperature of $T_{g}=300 \mathrm{~K}$, Table II shows various ranges of satellite altitudes and their effect on an instrument measured pressure. The orbits are chosen to be circular for simplicity, and the NRLMSISE-00 model at average thermospheric conditions (solar average) is used for generating thermospheric quantities of density and temperature with altitude.

From Table II, the altitude limits corresponding to the pressure range of Fig. 6 are $159 \mathrm{~km}$ and $294 \mathrm{~km}$. To investigate the instrument performance at higher altitudes, we use our test pressure measurements, invert them based on the ram effect to ambient pressures, and extrapolate from the linear fits. Using the data shown in Fig. 6 with an emission current value of $100 \mu \mathrm{A}$ and $\left.P_{g}\right|_{600 \mathrm{~km}}$ from Table II results in an MCP detector current of $\sim 740 \mathrm{pA}$. This is roughly a factor of 15 above a typical electrometer noise floor. ${ }^{6}$ Thus the signal to noise ratio is sufficient to allow good measurements.

It is therefore not unreasonable to expect successful operation of the instrument at this altitude. It should also be noted that these altitudes border on the exosphere, depending on solar activity. At altitudes above the exobase (nominally near $600 \mathrm{~km}$ ), the neutral particles follow ballistic trajectories, so it is inappropriate to describe them as having a bulk flow or

TABLE II. Ram effect on pressure measurements.

\begin{tabular}{lccc}
\hline \hline Significance & $\begin{array}{c}\text { Satellite } \\
\text { altitude }(\mathrm{km})\end{array}$ & $\begin{array}{c}P_{0} \\
(\mathrm{mPa})\end{array}$ & $\begin{array}{c}P_{g} \\
(\mathrm{mPa})\end{array}$ \\
\hline Figure 6, minimum altitude & 159 & 0.269 & 7.81 \\
Figure 6, maximum altitude & 294 & $6.04 \times 10^{-3}$ & 0.110 \\
Maximum claimed altitude & 600 & $3.48 \times 10^{-5}$ & $6.06 \times 10^{-4}$ \\
\hline \hline
\end{tabular}


wind direction. The REDD instrument described herein is not well suited to making neutral measurements of velocity at these altitudes but is instead designed to measure parameters related to a component of the neutral wind in the lower altitude range where collisional coupling between ions and neutrals is important to the ionospheric and thermospheric dynamics. In practice, a rigorous calibration of a flight instrument would be carried out and used in place of any theoretical form which would compensate for any unforeseen systematic biases and obtain true measurements.

The behavior in Fig. 6 is consistent over 30 independent trials. One plausible explanation for deviation from the fits is related to the volume of the effective region within which ionization is occurring inside the ionization chamber. At low pressures, there is essentially a higher density neutral film or boundary layer near surfaces due to outgassing. Ionization occurring near the surfaces in the chamber may lead to either an increased likelihood that the ions will be successfully drawn out by the grid bias or that they will be collected by the chamber walls, depending on the surface location. Another possible explanation is space charge effects since the varying ionization efficiency as a function of pressure modifies the shielding effects and may lead to more complex behavior. It should be noted that these described possibilities are not of concern for normal operation in a flowing medium with significant kinetic energy since the high ram velocity increases the flux of neutrals and ions into the RPA gridstack.

Functional validation of the REDD RPA subsystem using an external ion source is shown in Fig. 7. To reiterate, this ion beam source is used to replicate LEO energy ions that would be produced by the REDD internal ionization source. It is worth noting that the measured $I V$ characteristic is not consistent with that which would be seen under orbital conditions because the output of the external ion source in our tests is not a Maxwellian energy distribution. Ions in the source are necessarily born within a range of potential energies dependent on the internal biases of the source. These ions have long mean free paths relative to the distance from the source to the detector, so we do not expect a Maxwellian energy distribution. This is apparent in the figure due to the immediate suppression of the detector current as the retarding potential is increased. For a true Maxwellian distribution of input gas, the low voltage region of the $I V$ would be nearly flat, as shown by Fanelli et al. ${ }^{6}$ The apparent inflection points and non-Maxwellian form of the $I V$ characteristic are due to the geometry of the external ionization source. The main result shown in Fig. 7 is successful functionality of the REDD RPA to suppress ion current in accordance with our expectations.

\section{CONCLUSION}

In the work presented here, we have demonstrated the three primary subsystems of the REDD instrument: the plasma extraction system, the ionization source and detection system, and the retarding potential analyzer (RPA) energy diagnostic system under LEO like orbit vacuum pressures and particle energies. The design improvements made to this new generation of the RWS enable more widespread implementation in the small satellite community and subsequent broadening of our sample coverage of these critical geophysical parameters. The final step in validating REDD is to run all the subsystems simultaneously in a vacuum system that contains a calibrated neutral beam source, preferably one that can flow oxygen gas at speeds of several thousand meters per second. While such a test is not possible using our current laboratory facilities, we are actively working to perform such tests using systems available in other laboratories. Results of end-to-end validation testing in this system, including comparisons to more sophisticated numerical simulations, will be reported in a future publication.

\section{ACKNOWLEDGMENTS}

This research was supported by Air Force Research Lab small business innovative research (SBIR) Grant No. AF121-066 and carried out in collaboration with Dr. Ted Beach and Dr. Odile Clavier from Creare LLC. The authors thank Dr. Cheryl Huang, Dr. John Ballenthin, and Dr. Patrick Roddy for supporting and reviewing this project at AFRL.

${ }^{1}$ K. Groves, S. Basu, E. Weber, M. Smitham, H. Kuenzler, C. Valladares, R. Sheehan, E. MacKenzie, J. Secan, P. Ning et al., Radio Sci. 32, 2047 (1997).

${ }^{2}$ D. Hysell, J. Atmos. Sol.-Terr. Phys. 62, 1037 (2000).

${ }^{3}$ G. Earle, J. Klenzing, P. Roddy, W. Macaulay, M. Perdue, and E. Patrick, Rev. Sci. Instrum. 78, 114501 (2007).

${ }^{4}$ W. C. Knudsen, J. Geophys. Res. 71, 4669, https://doi.org/10.1029/ jz071i019p04669 (1966).

${ }^{5}$ E. Whipple, "The ion trap results in 'Exploration of the upper atmosphere with the help of the third Soviet Sputnik,"” Proc. Inst. Radio Eng. 47, 2023 (1959).

${ }^{6}$ L. Fanelli, S. Noel, G. Earle, C. Fish, R. Davidson, R. Robertson, P. Marquis, V. Garg, N. Somasundaram, L. Kordella et al., Rev. Sci. Instrum. 86, 124501 (2015).

${ }^{7}$ I. Brodie and C. Spindt, Adv. Electron. Electron Phys. 83, 1 (1992).

${ }^{8}$ P. A. Roddy, "Spatial and temporal structuring of nighttime intermediate layers above Wallops Island," Ph.D. thesis, University of Texas at Dallas, 2005.

${ }^{9}$ J. Westerhoff, G. Earle, R. Bishop, G. R. Swenson, S. Vadas, J. Clemmons, R. Davidson, L. Fanelli, C. Fish, V. Garg et al., Adv. Space Res. 56, 1413 (2015).

${ }^{10}$ S. Debchoudhury and G. Earle, Phys. Plasmas 24, 042902 (2017).

${ }^{11}$ R. H. Fowler and L. Nordheim, Proc. R. Soc. London, Ser. A 119, 173-181 (1928).

${ }^{12}$ D. Zuccaro and B. Holt, J. Geophys. Res.: Space Phys. 87, 8327, https://doi.org/10.1029/ja087ia10p08327 (1982).

${ }^{13}$ I. Brodie and P. R. Schwoebel, Proc. IEEE 82, 1006 (1994).

${ }^{14}$ G. N. Fursey, Field Emission in Vacuum Microelectronics (Springer Science \& Business Media, 2007).

${ }^{15}$ W. Hanson, U. Ponzi, C. Arduini, and M. Di Ruscio, J. Astronaut. Sci. 40, 429 (1992).

${ }^{16}$ C. Spindt, C. Holland, A. Rosengreen, and I. Brodie, IEEE Trans. Electron Devices 38, 2355 (1991).

${ }^{17}$ Performance validation of the REDD ambient plasma extraction plates and the REDD RPA is achieved through vacuum chamber testing with an externally generated ion beam source. Though ionospheric electron thermal energies are only on the order of $200 \mu \mathrm{V}$, the ram energy component of LEO ions seen by a high velocity satellite is on the order of $10 \mathrm{~V}$. The external ion beam source is therefore configured to output LEO-like ion energy magnitudes within a distribution of $0-12 \mathrm{eV}$. This is consistent with sweep ranges of traditional LEO RPA designs. ${ }^{6}$ 